\title{
Meta-analysis of the benefit of sitagliptin treatment in patients with type 2 diabetes complicated with incipient nephropathy
}

\author{
WEI LIU ${ }^{1}$, JIANGYI YU ${ }^{1,2}$, QIANHUA YAN ${ }^{2}$, LIJUAN WANG ${ }^{2}$, NAN LI $^{1}$ and WEI XIONG ${ }^{1}$ \\ ${ }^{1}$ The First Clinical Medical College, Nanjing University of Chinese Medicine; ${ }^{2}$ Department of Endocrinology and \\ Metabolism, Jiangsu Province Hospital of Traditional Chinese Medicine, Affiliated Hospital of \\ Nanjing University of Chinese Medicine, Nanjing, Jiangsu 210023, P.R. China
}

Received January 25, 2018; Accepted July 2, 2018

DOI: $10.3892 /$ etm.2018.6449

\begin{abstract}
The purpose of this meta-analysis was to evaluate the evidence of the clinical efficacy and safety of sitagliptin in diabetic patients with incipient nephropathy. PubMed, Ovid, Cochrane library, Chinese National Knowledge Infrastructure, and Wanfang databases were searched in September 2017 to identify randomized controlled trials (RCTs) of sitagliptin in diabetic patients with incipient nephropathy. Study selection, data extraction and study quality assessment were performed independently by two investigators, while disagreements were resolved by a third reviewer. The treatment effect was estimated by calculating the mean difference (MD) or standard mean difference (SMD). Heterogeneity was assessed with the $\chi^{2}$ and $\mathrm{I}^{2}$ tests. Additionally, risk of bias graphs and summaries were used to assess the quality of the included trials. Thirteen RCTs were included in this review; their results suggested that sitagliptin has obvious advantages in lowering the 24-hour urinary albumin excretion [MD, $-25.71 ; 95 \%$ confidence interval (CI), -30.75 to $-20.66 ; \mathrm{P}<0.00001]$, serum cystatin $\mathrm{C}(\mathrm{MD},-0.59$; 95\% CI, -0.64 to $-0.54 ; \mathrm{P}<0.00001$ ), inflammation (MD, -0.81 ; $95 \% \mathrm{CI},-1.20$ to $-0.42 ; \mathrm{P}<0.0001$ ), and total cholesterol (MD, $-0.13 ; 95 \%$ CI, -0.22 to $-0.03 ; \mathrm{P}=0.009)$. However, sitagliptin did not appear to influence serum creatinine, fasting blood glucose, postprandial blood glucose, glycosylated hemoglobin A1c, or triglyceride levels, although these results may have been influenced by biases in the included trials. The most common adverse effects of sitagliptin were gastrointestinal tract reaction and hypoglycemia, although these symptoms resolved quickly. Sitagliptin appears to be effective in reducing proteinuria, ameliorating renal function, and producing an anti-inflammatory effect in patients with early-stage diabetic
\end{abstract}

Correspondence to: Professor Jiangyi Yu, The First Clinical Medical College, Nanjing University of Chinese Medicine, 138 Xian Lin Avenue, Nanjing, Jiangsu 210023, P.R. China

E-mail: yujiangyi2017@163.com

Key words: sitagliptin, type 2 incipient diabetic nephropathy, meta-analysis nephropathy. The present analysis provides important guidance for the clinical application of sitagliptin.

\section{Introduction}

Type 2 diabetes mellitus (DM) is defined as a metabolic disorder in which patients become hyperglycemic from defects in insulin secretion or action, as well as from the hyperglucagonemia that ensues (1). Diabetic nephropathy (DN) is one of the major chronic microvascular complications of DM (2), and occurs in $20-40 \%$ of all patients with type $2 \mathrm{DM}(2,3)$. DN is caused by rising blood glucose and hypertension, obesity, oxidative stress, and unmitigated inflammation. Repeated or chronic insult to the kidneys leads to irreversible fibrotic damage of the glomeruli (glomerulosclerosis) and kidney tubules (tubulointerstitial fibrosis), and ultimately leads to end-stage renal disease (ESRD) (4). Due to these potential complications, DN can also cause a significant financial burden. Therefore, early diagnosis and management are critical.

Diabetes with microalbuminuria (30 mg/day) is an early clinical sign of DN; this subsequently progresses to macroalbuminuria (>300 mg/day) and a decline in glomerular filtration rate (5). Over the past 20 years, therapies have significantly progressed beyond those that primarily reduce glucose levels; however, these therapies are also associated with unwanted side effects, such as weight gain. More recently developed drug classes can effectively improve glycemia and promote weight neutrality or even weight loss (6). Dipeptidyl peptidase-4 (DPP-4) inhibitors belong to these more recent drug classes.

DPP-4 inhibitors are a class of oral antihyperglycemic treatments for type 2 diabetes. They include sitagliptin, saxagliptin, vildagliptin, linagliptin, and teneligliptin. Sitagliptin, the first DPP-4 inhibitor on the market, was approved in 2006 for patients with type 2 DM (6), and has been widely used for the treatment of both DM and its complications, particularly DN. A recent study showed that DPP-4 inhibition in conjunction with angiotensin II type 1 receptor blockade produced enhanced renoprotective effects in patients with DN (7). However, whether sitagliptin has renoprotective properties remains controversial. Hattori's (8) study suggested that sitagliptin reduces albuminuria, which is dependent on satisfactory control of blood sugar. However, other groups discount the presence of a significant relationship between the 
change in the UACR and HbAlc, and claim that sitagliptin reduces blood pressure by increasing sodium diuresis (9-12). Sitagliptin increases the level of glucagon-like polypeptide (GLP)-1, GLP-1 decreases salt intake and increases urinary salt excretion by directly inhibiting the $\mathrm{Na}^{+} / \mathrm{H}^{+}$exchange at the proximal tubular cells $(11,12)$. The abovementioned studies did not measure sodium diuresis and the sample sizes of the sitagliptin-treated cohorts were too small to be generalized; for example, Hattori's (8) study included only 36 subjects. Furthermore, some investigations (such as that of Kawasaki et al (9)) were non-randomized and uncontrolled observation studies. Thus, whether sitagliptin truly provides a benefit remains unconfirmed. To that end, we performed a systematic review of several randomized controlled trials (RCTs) to investigate the effect of sitagliptin in patients with type 2 diabetes that also had incipient nephropathy. Moreover, we deem that sitagliptin truly has renoprotective effect for patients with early-stage DN.

\section{Materials and methods}

Search strategy. Electronic searches of the PubMed, OVID, Cochrane library, Chinese National Knowledge Infrastructure (CNKI) and Wanfang databases were performed independently by two investigators in September 2017. The search terms were: ('Sitagliptin phosphate' or 'sitagliptin' or 'sitagliptin phosphate monohydrate') and ('diabetic nephropathies' or 'diabetic nephropathy' or 'diabetic kidney diseases' or 'diabetic complications'). We filtered the publications by titles, abstracts and keywords, and assessed the full-text versions while employing our inclusion and exclusion criteria (described below). The publication languages were restricted to English and Chinese. All clinical studies except case reports were chosen for our analysis. The first reports' bibliographies, as well as references from selected studies, were also searched for other relevant publications.

Selection criteria. We collected all relevant articles focusing on the relationship between sitagliptin and renal function index. The inclusion criteria were as follows: i) RCTs that compared sitagliptin to a control group that received routine treatment, or to drugs other than DPP-4 inhibitors; ii) all participants were type 2 diabetes patients with incipient nephropathy, with persistent microalbuminuria (incipient DN) defined as a urinary albumin-to-creatinine ratio between 30 and $300 \mathrm{mg} / \mathrm{g}$ in two morning spot urine collections sustained over 12 weeks (2); iii) renal function outcomes were reported in the studies; and iv) the study duration was longer than eight weeks. The following exclusion criteria were applied: i) Articles that had no information on renal function or the biochemical index of type $2 \mathrm{DN}$; ii) case reports, letters, reviews, expert opinion, conference abstracts, editorials, and non-English and non-Chinese language papers; and iii) all articles using cell lines and/or in vitrolex vivo studies.

Data extraction. The qualified studies were reviewed by two investigators independently; differences in interpretation were resolved by consensus. The following information was recorded for each study: General data, patient information, test drug information, course of treatment, and outcome data. The two researchers identified the articles that satisfied the selection criteria. Data heterogeneity was assessed to determine whether the studies could be analyzed. Study characteristics and clinical examination data were generalized and are described in table format.

Statistical analysis. Quantitative and qualitative analyses were performed on corrected data. The RevMan v.5.3 software was downloaded from the Cochrane collaboration website and used for meta-analysis. The clinical and methodological heterogeneity of the included studies were analyzed using the $\chi^{2}$ and $\mathrm{I}^{2}$ tests. The fixed-effects model was used when $\mathrm{P}>0.1$ and $\mathrm{I}^{2}<50 \%$, and each study showed acceptable heterogeneity. In cases where $\mathrm{P}<0.1$ and $\mathrm{I}^{2}>50 \%$, and the study showed essential heterogeneity, we performed subgroup or sensitivity analysis. However, when many confounding factors appeared in the studies, the random-effects model was chosen regardless of the $\mathrm{P}$ and $\mathrm{I}^{2}$ values, according to the Cochrane Handbook (13). The mean difference (MD) or standard mean difference (SMD) and $95 \%$ confidence intervals (CIs) were used to compare continuous variables; risk ratios and $95 \%$ CIs were used to compare dichotomous variables (13). Whenever heterogeneity was significant, we sought to identify its source using the study-by-study exclusion method. $\mathrm{P}<0.05$ was considered to indicate a statistically significant difference. Egger's test and funnel plots were used to detect publication bias.

Assessments of quality of evidence. Two investigators independently assessed the risk of bias as recommended by the Cochrane Handbook for Systematic Reviews of Interventions (13). Disagreements were resolved by a third reviewer. The quality appraisal of the literature included: Random sequence generation, allocation concealment, blinding of participants and personnel, blinding of outcome assessment, incomplete outcome data, selective reporting, and other biases. Articles that had clearly described details and met or surpassed the quality criteria were defined as low risk; otherwise, they were deemed high-risk. Equivocal articles in terms of quality criteria were deemed to be of unclear risk.

\section{Results}

Study characteristics. We initially collected 85 articles from the PubMed, OVID, the Cochrane library, CNKI, and Wanfang databases. Thirty full-text studies were extracted for more detailed assessment, and were filtered via their titles and abstracts for final eligibility assessment. Ten crossover trials without control groups were excluded, as were seven trials that lacked renal function marker analysis. Eventually, 13 publications that satisfied the inclusion criteria were selected for this meta-analysis. The article search process and study selection are shown in Fig. 1.

Of the total 942 patients included, 481 belonged to treatment groups, while 461 belonged to control groups receiving routine treatment such as controlled diet and exercise therapy, antihypertensive drugs, or other antihyperglycemic treatments excluding DPP-4 inhibitors. The treatment groups included sitagliptin combined with routine treatment, antihypertensive drugs, or other antihyperglycemic treatment. The doses 


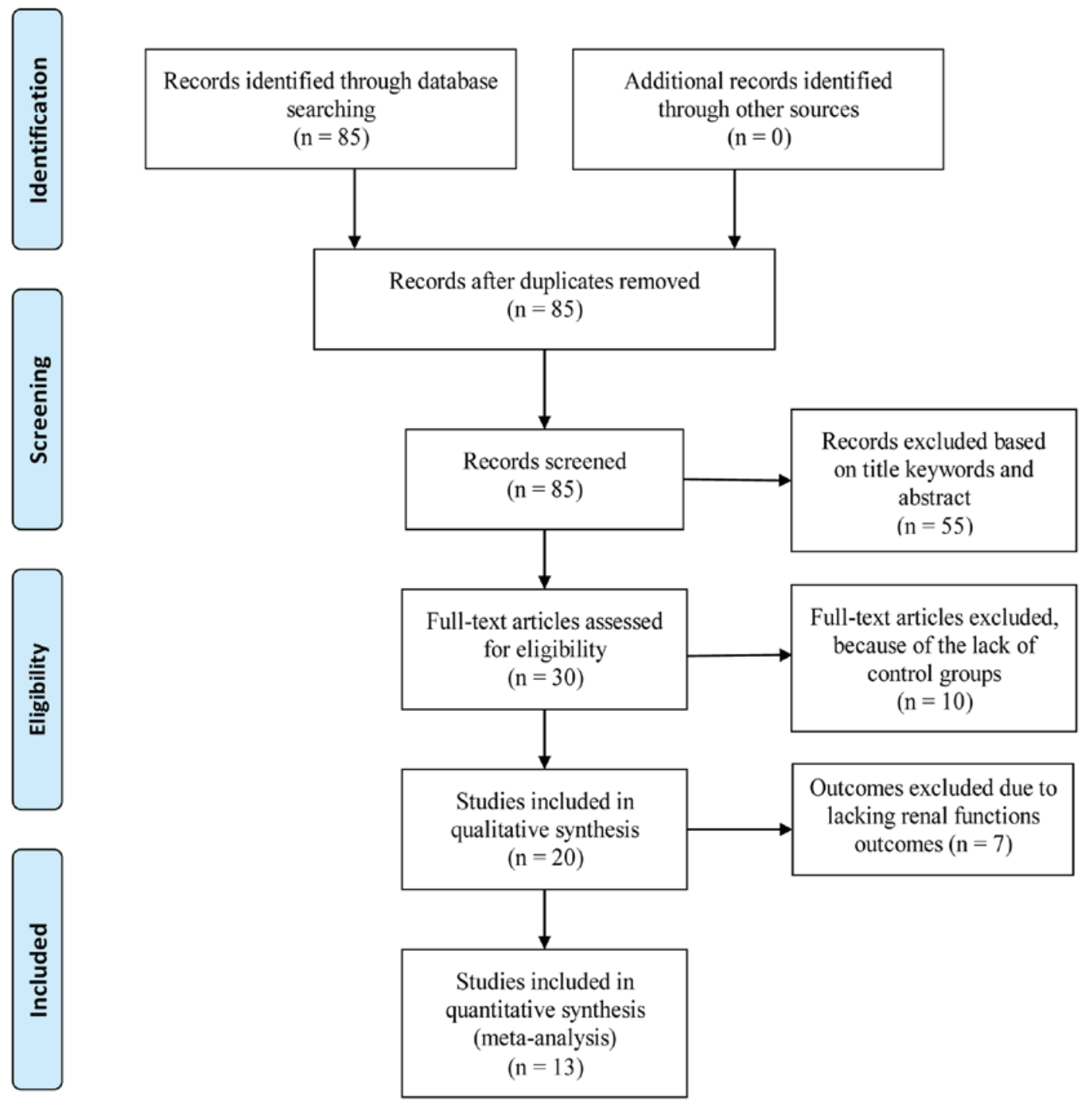

Figure 1. Study selection flowchart.

of sitagliptin used in the included trials differed slightly. Sitagliptin intake was generally $100 \mathrm{mg}$ per day; only one trial used $50 \mathrm{mg}$ per day (14). Among the 13 trials, 12 adopted a two-armed parallel group design while one (Zhang et al (15)) adopted a three-armed group design. The durations of interventions in the diabetes trials were different, ranging from eight to 24 weeks. Six studies lasted 24 weeks, one lasted eight weeks, and the remainder lasted 12 weeks. Basic information about the involved studies is shown in Table I.

Risk of bias. The risk of bias assessments are shown in Figs. 2 and 3. All trials were randomly designed, but eight (16-23) were judged to have unclear risks of bias owing to allocation concealment.

Blinding of participants, personnel, and outcome assessors were not undertaken in three studies $(15,21,22)$. Additionally, it was not clear whether the participants and personnel were blinded in eight trials (16,18-20,23-26). Furthermore, it was unclear if the outcome assessors were blinded in eight of the studies $(16-20,23,24,26)$. Attrition bias was ambiguous in eight of the trials $(15-18,21-23,26)$; however, all trials showed a low risk of reporting bias or other biases.

\section{Effect of interventions}

Effect on proteinuria and renal function. Five trials $(15,20,22,24,25)$ investigated the effect of sitagliptin on quantitative 24-hour urinary total protein (UTP) test results (Fig. 4A). The sitagliptin and control groups comprised 170 and 148 patients, respectively. No significant heterogeneity was found between the results $\left(\chi^{2}=7.02 ; \mathrm{I}^{2}=43 \%, \mathrm{P}=0.14\right)$; hence, a fixed-effect model was used for statistical analysis. The 24-h UTP was lower in the treatment group than in the control group $(\mathrm{MD}=-25.71,95 \% \mathrm{CI}-30.75$ to -20.66 , $\mathrm{P}<0.00001)$. Three trials (19-21) were used to compare serum cystatin C (CysC) levels between the two groups (Fig. 4B). The treatment group included 92 patients and the control group included 95 patients. No significant heterogeneity was found between the two groups $\left(\chi^{2}=2.26 ; \mathrm{I}^{2}=11 \%, \mathrm{P}=0.32\right)$. Patients receiving sitagliptin had better CysC levels than the control group $(\mathrm{MD}=-0.59,95 \% \mathrm{CI}-0.64$ to-0.54, $\mathrm{P}<0.00001)$. Three trials reported serum creatinine (SCr) levels $(20,23,25)$ with 106 patients in each of the treatment and control groups. These trials showed homogeneity in the consistency of their results $\left(\chi^{2}=1.00 ; \mathrm{I}^{2}=0 \%, \mathrm{P}=0.61\right)$. Therefore, a fixed-effect model was selected for statistical analysis, which revealed 
Table I. Characteristics of the included studies.

\begin{tabular}{|c|c|c|c|c|c|c|c|}
\hline Stydy, year & $\begin{array}{l}\text { Sample } \\
\text { size }\end{array}$ & $\begin{array}{l}\text { Testing } \\
\text { scheme }\end{array}$ & Test group & Control group & $\begin{array}{l}\text { Duration } \\
\text { (weeks) }\end{array}$ & Outcomes & (Refs.) \\
\hline Mori et al, 2014 & $41 / 44$ & RAN & SIT (50 mg qd) Plus RT & RT & 24 & ACFGH & (14) \\
\hline Zhang et al, 2016 & $29 / 29$ & UNK & SIT (100 mg qd) Plus IRB & IRB (150 mg qd) & 12 & GI & (15) \\
\hline Hu et al, 2016 & $80 / 80$ & RAN & SIT (100 mg qd) Plus RT & RT & 12 & $\mathrm{ABCH}$ & (16) \\
\hline Huang et al, 2016 & $30 / 30$ & RAN & SIT (100 mg qd) Plus GLI & GLI (30 mg qd) & 12 & ABCGJ & (17) \\
\hline Jin et al, 2016 & $40 / 40$ & RAN & SIT (100 mg qd) Plus RT & $\mathrm{RT}$ & 24 & $\mathrm{ACFJ}$ & (18) \\
\hline Lan et al, 2016 & $24 / 24$ & RAN & SIT (100 mg qd) Plus RT & RT & 24 & $\mathrm{ABCL}$ & (19) \\
\hline Yang et al, 2015 & $36 / 36$ & RAN & SIT (100 mg qd) Plus RT & RT & 24 & ACDEILK & $(20)$ \\
\hline Ying et al, 2016 & $32 / 35$ & UNK & SIT (100 mg qd) Plus RT & RT & 12 & ACL & (21) \\
\hline Zhao et al, 2014 & $35 / 13$ & UNK & SIT (100 mg qd) Plus VAL & $\operatorname{VAL}(80 \mathrm{mg}$ qd) & 24 & CI & $(22)$ \\
\hline Huang et al, 2016 & $30 / 30$ & RAN & SIT (100 mg qd) Plus RT & RT & 12 & ACDEFK & $(23)$ \\
\hline Wang et al, 2015 & $30 / 30$ & RAN & SIT (100 mg qd) Plus Insulin & INS & 8 & ADEIJ & $(24)$ \\
\hline Han et al, 2015 & $40 / 40$ & RAN & SIT (100 mg qd) Plus GLI & GLI (30 mg qd) & 12 & ABCDEFIK & $(25)$ \\
\hline Hао et al, 2015 & $34 / 30$ & RAN & SIT (100 mg qd) Plus RT & RT & 24 & ACDEFGH & (26) \\
\hline
\end{tabular}

RNA, randomized controlled trial; UNK, unknown; SIT, sitagliptin; RT, routine treatment; GLI, gliclazide; VAL, valsartan; IRB, irbesartan; INS, insulin; A, fasting blood glucose; B, postprandial blood glucose; C, glycosylated hemoglobin; D, total cholesterol; E, triglyceride; F, low density lipoprotein cholesterol; G, urine albumin-to-creatinine ratio; H, estimated glomerular filtration rate; I, 24-hour urinary protein quantitative; J, C-reactive protein; K, serum creatinine; L, serum cystatin $\mathrm{C}$.

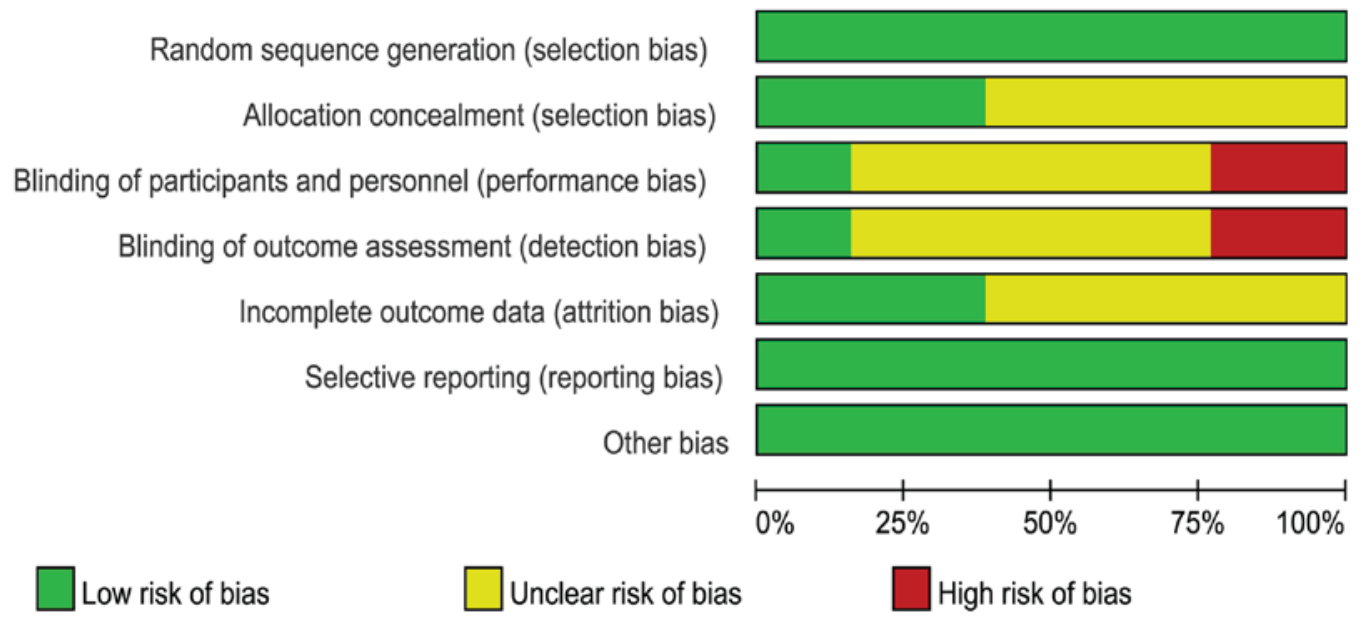

Figure 2. Assessed risks of bias in various categories across all the included studies.

no difference between the sitagliptin and control groups in terms of changes in SCr levels (MD $=-1.07,95 \%$ CI-3.52 to $1.37, \mathrm{P}=0.39$; Fig. 4C). Overall, however, the results suggested that sitagliptin reduced proteinuria, thus ameliorating renal function.

Effect on improving inflammation. Three trials $(17,18,24)$ compared C-reactive protein (CRP) levels between the experimental and control groups (100 patients each); no significant heterogeneity was observed $\left(\chi^{2}=0.47 ; \mathrm{I}^{2}=0 \%, \mathrm{P}=0.79\right.$; Fig. 5) and a fixed-effect model was used for statistical analysis. The sitagliptin group expressed lower CRP levels than the control group (MD=-0.81; 95\% CI-1.20 to $-0.42 ; \mathrm{P}<0.0001$; Fig. 5), indicating that sitagliptin exhibits anti-inflammatory effects in patients with DN.
Effect on blood glucose and glycosylated hemoglobin. Eleven trials (14,16-21,23-26) investigated the effect of sitagliptin on fasting blood glucose (FBG) levels. The sitagliptin and control groups comprised 417 and 419 patients, respectively. The statistical heterogeneity of the FBG data was unacceptable $\left(\chi^{2}=17.10 ; \mathrm{I}^{2}=42 \%, \mathrm{P}=0.07\right)$; therefore, we used the random-effect model for meta-analysis. The pooled result showed no difference between the sitagliptin and control groups $(\mathrm{SMD}=-0.06 ; 95 \% \mathrm{CI}-0.20$ to $0.07 ; \mathrm{P}=0.36$; Fig. 6A).

Four trials $(16,19,23,25)$ investigated the effect of sitagliptin on postprandial blood glucose (PBG) with 174 in each of the treatment and control groups; there was no significant heterogeneity $\left(\chi^{2}=1.37 ; \mathrm{I}^{2}=0 \%, \mathrm{P}=0.71\right)$ and a fixed-effect model was used. However, no differences in FBG between the 


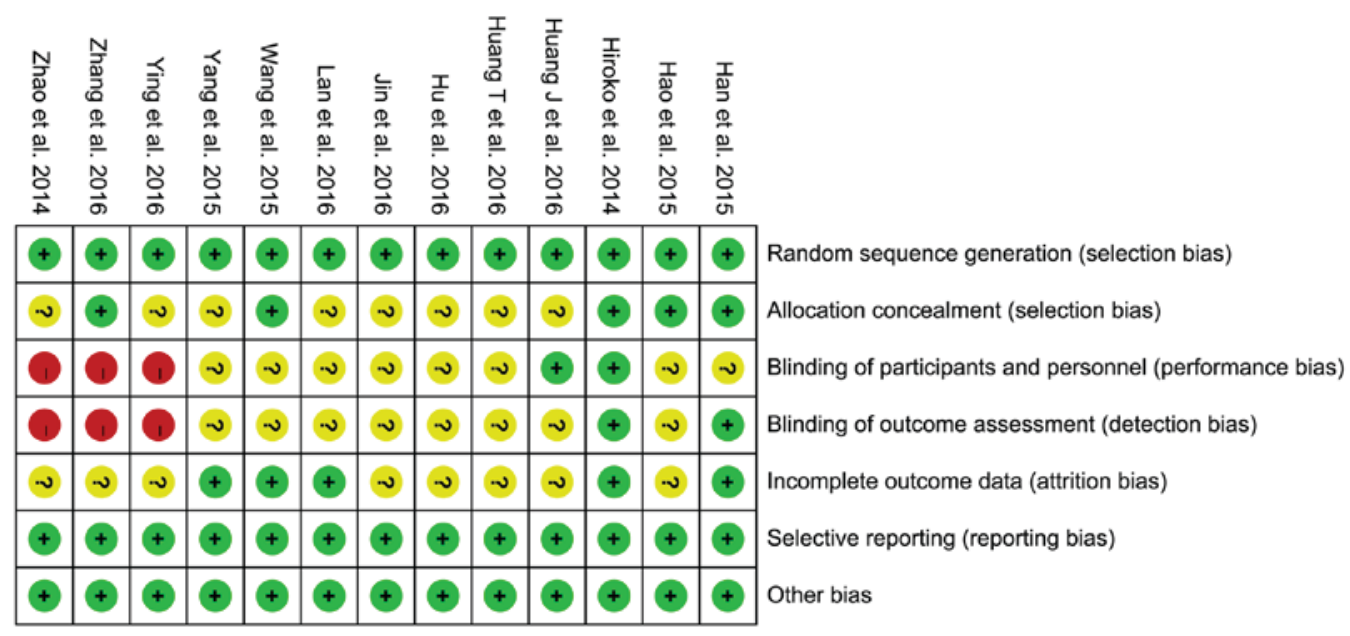

Figure 3. The risk of bias per category for each included study.

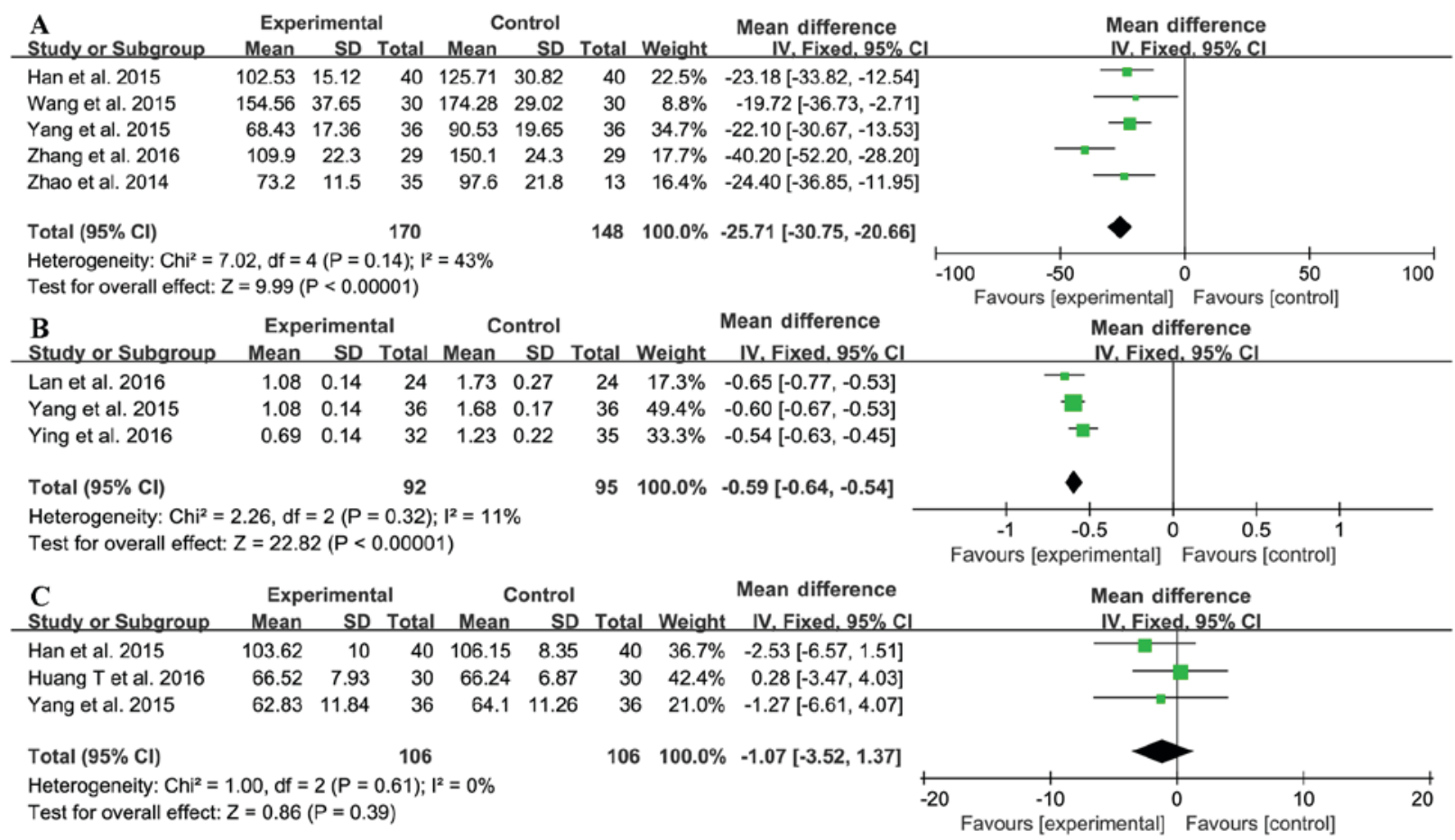

Figure 4. Forest plot showing the effect of sitagliptin in patients with DN. (A) 24-h urinary total protein; (B) serum cystatin C; (C) serum creatinine. DN, diabetic nephropathy; SD, standard deviation; CI, confidence interval; df, degrees of freedom.

two groups were detected ( $\mathrm{MD}=-0.23 ; 95 \% \mathrm{CI}-0.52$ to 0.06 ; $\mathrm{P}=0.12$; Fig. 6B).

As shown in Fig. 6C, 11 trials $(14,16-23,25,26)$ investigated the effect of sitagliptin on HbAlc; the heterogeneity was acceptable $\left(\chi^{2}=14.03 ; I^{2}=29 \%, P=0.17\right)$, and therefore the fixed-effect model was used. Sitagliptin did not influence HbA1c levels ( $\mathrm{MD}=-0.04 ; 95 \% \mathrm{CI}-0.10$ to 0.03 ; $\mathrm{P}=0.30$ ); hence, it is unclear if sitagliptin decreases blood glucose levels.

Effect on blood lipids. Five trials (20,23-26) investigated total cholesterol (TC) and triglyceride (TG) levels (Fig. 7), with 170 subjects in the treatment group and 166 in the control group. There was no significant heterogeneity between the TC and TG results (TC: $\chi^{2}=5.31, \mathrm{I}^{2}=25 \%, \mathrm{P}=0.26$; TG: $\chi^{2}=5.93, \mathrm{I}^{2}=33 \%$,
$\mathrm{P}=0.20$ ), so the fixed-effect model was used for meta-analysis. Sitagliptin treatment reduced the level of TC $(\mathrm{MD}=-0.13,95 \%$ CI-0.22 to-0.03, $\mathrm{P}=0.009)$, but not TG (MD=-0.01,95\% CI-0.07 to $0.05, \mathrm{P}=0.73$; Fig. 7). These data showed that sitagliptin does affect blood lipid levels.

Adverse events. Only five trials $(15,17,21,22,25)$ investigated adverse events, two of which $(17,21)$ reported no such occurrences. Of the remaining three trials, one (25) found that patients in the treatment group presented with hypoglycemia $(\mathrm{n}=2)$, dizziness $(\mathrm{n}=2)$, and gastrointestinal tract reaction $(n=2)$; the same conditions were experienced in patients in their control groups $(n=3, n=1$, and $n=2$, respectively). Another trial (15) reported a slight cough $(n=1)$ in the control group 


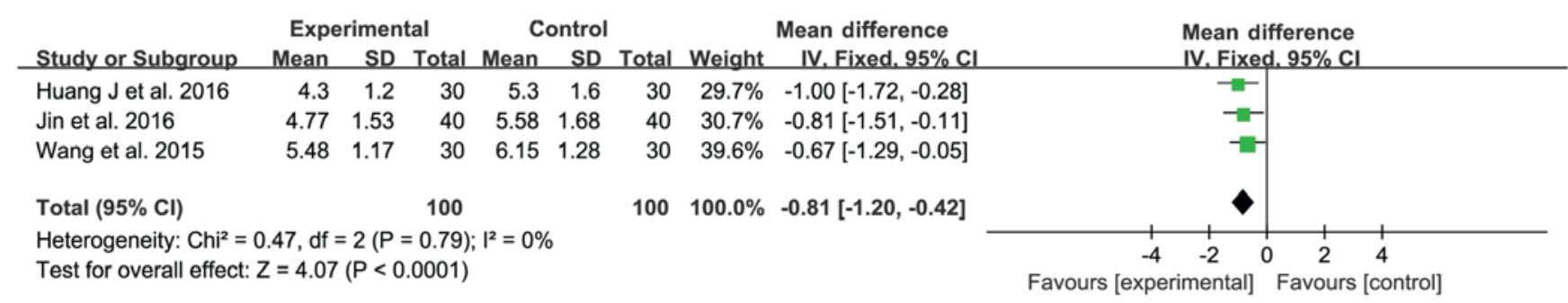

Figure 5. Forest plot showing the effects of sitagliptin treatment in patients with DN. DN, diabetic nephropathy; SD, standard deviation; CI, confidence interval; df, degrees of freedom.

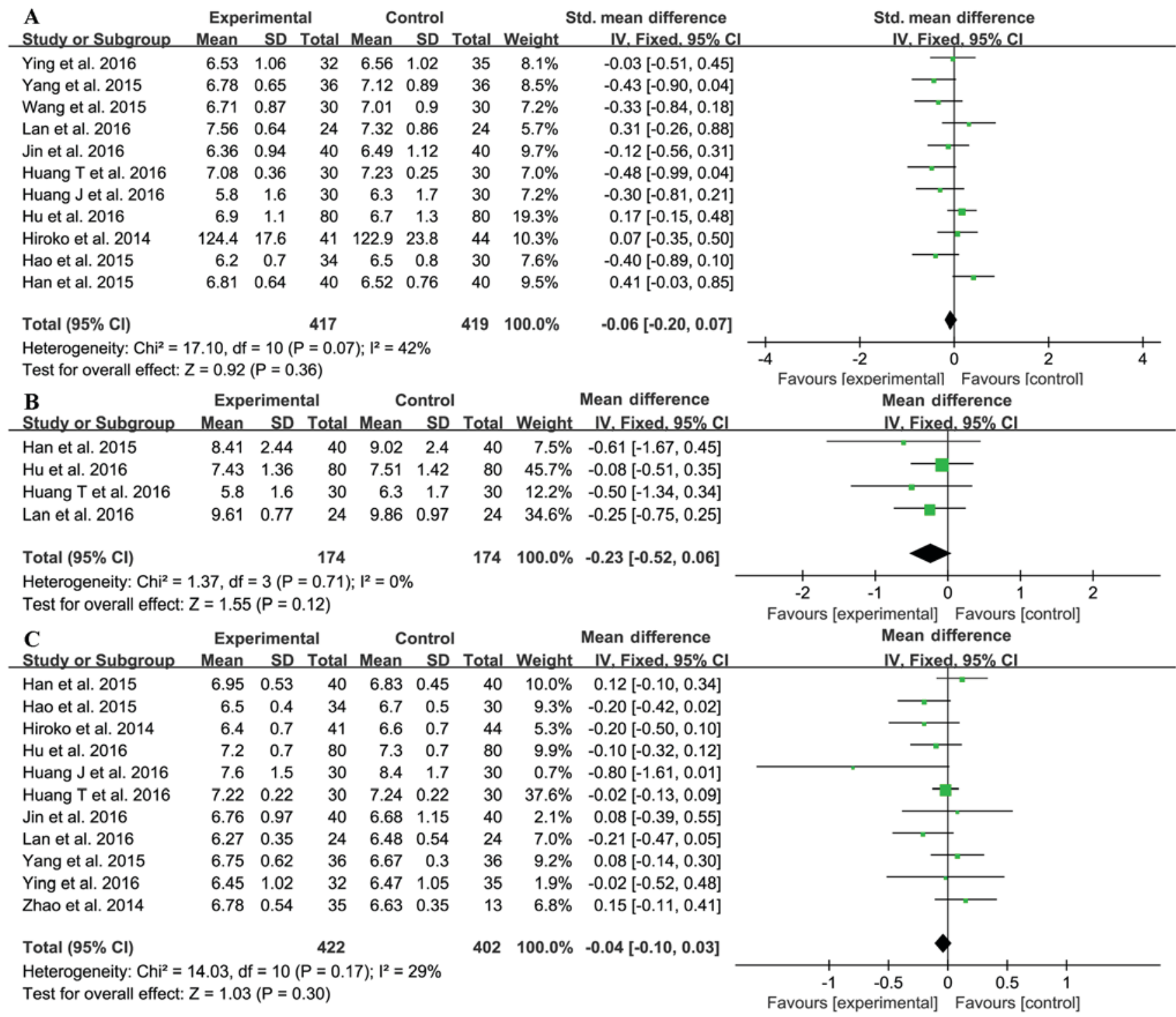

Figure 6. Forest plot showing the effect of sitagliptin for DN. (A) fasting blood glucose; (B) postprandial blood glucose; (C) glycosylated hemoglobin A1c. DN, diabetic nephropathy; SD, standard deviation; CI, confidence interval; df, degrees of freedom.

and gastrointestinal tract reaction $(\mathrm{n}=2)$ in the treatment group. The final trial (22) reported adverse effects without specifying their natures. However, there were no serious adverse events reported in any of these trials. The most common events were gastrointestinal tract reactions and hypoglycemia, but these symptoms resolved quickly.
Evaluation of publication bias. In the present study, funnel plots were used to identify publication bias. Asymmetry is observed in Fig. 8A and B; moreover, one study falls outside the $95 \%$ confidence interval (Fig. 8A). Egger's test of FBG $(\mathrm{P}=0.164)$ and HbAlc $(\mathrm{P}=0.941)$ showed that there was no publication bias in the studies included (Fig. 8A and B). 


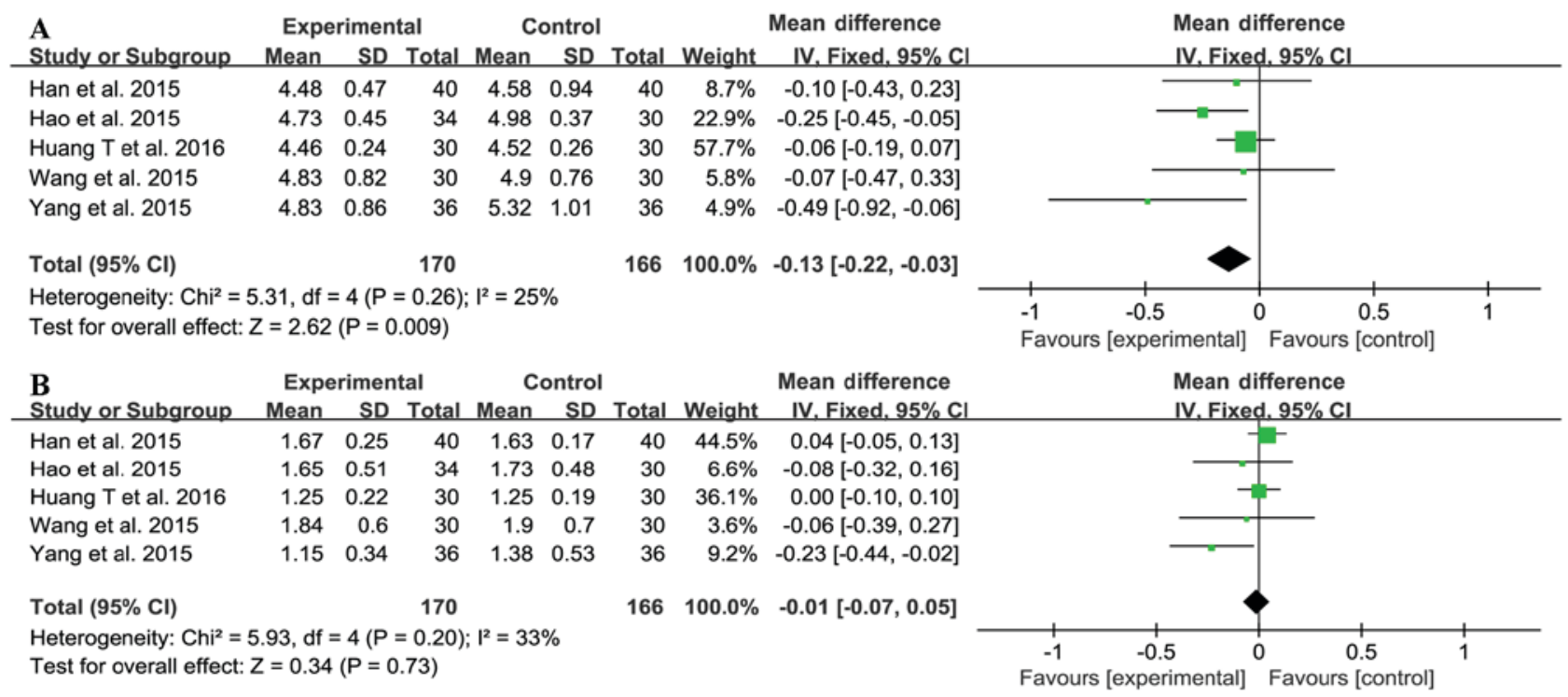

Figure 7. Forest plot showing the effect of sitagliptin for DN. (A) total cholesterol; (B) triglyceride. SD, standard deviation; CI, confidence interval; df, degrees of freedom; DN, diabetic nephropathy.
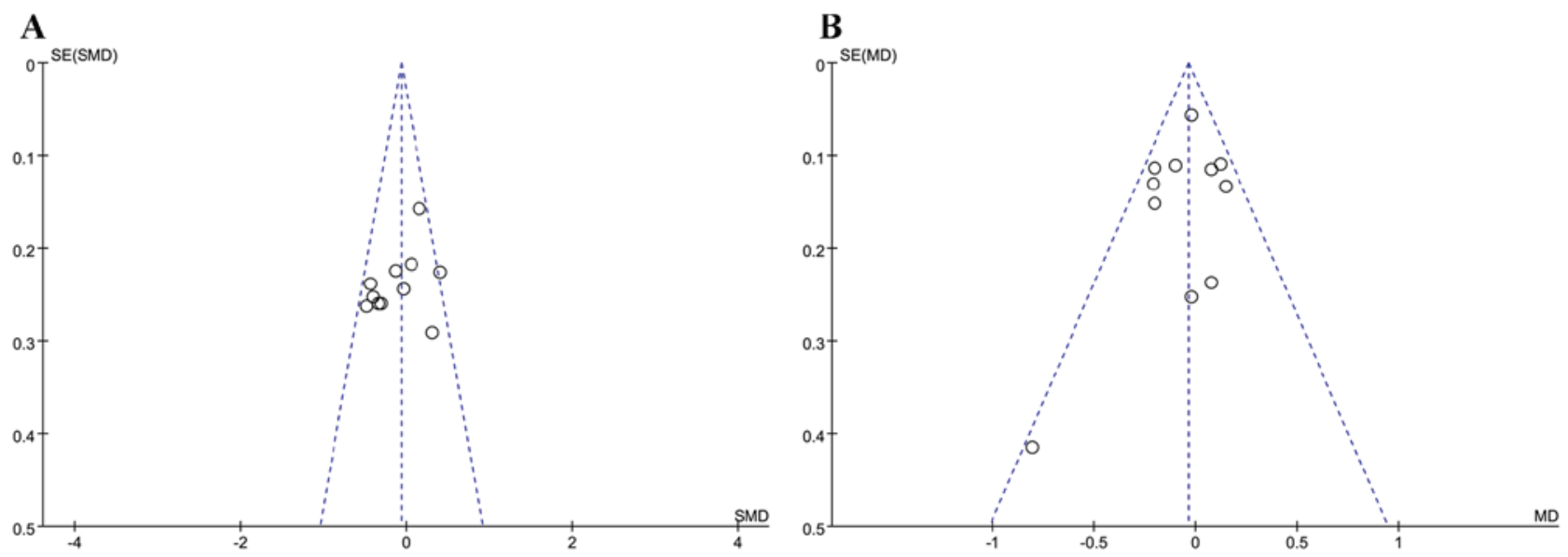

Figure 8. Funnel plot showing the effect of sitagliptin for DN. (A) fasting blood glucose; (B) glycosylated hemoglobin A1c. SD, standard deviation; CI, confidence interval; df, degrees of freedom; DN, diabetic nephropathy.

\section{Discussion}

DN treatment aims to reduce proteinuria. Angiotensin II receptor blockers (ARBs) are widely used for the improvement of proteinuria in patients with $\mathrm{DN}$, and provide more favorable outcomes especially in the incipient DN stage, as shown in the INNOVATION study $(27,28)$. However, ARBs cannot delay progression to ESRD, thus rendering renal replacement therapy inevitable. Therefore, determining whether sitagliptin exhibits a renoprotective effect is of great scientific value.

Our meta-analysis included 942 patients; most of the investigated trials adopted a randomized double-blind parallel control design. For assessing kidney function, 24-h UTP, CysC, and SCr were chosen as the ideal markers of kidney function because they are less influenced by age, sex, body mass, and inflammatory conditions $(29,30)$. Additionally, our data suggested that sitagliptin may have positive effects on lowering the 24-h UTP, CysC, and CRP. Although sitagliptin did not appear to affect $\mathrm{SCr}$, it still offers the benefit of reducing proteinuria, ameliorating renal function, and producing an anti-inflammatory response in patients with DN.

Importantly, TGF- $\beta_{1} / \mathrm{Smad} 3$ is known to mediate fibrosis in DN. A recent study found that sitagliptin prevented kidney injury, reduced albuminuria, and reduced inflammation by blocking the TGF- $\beta_{1} / \mathrm{Smad} 3$ signaling pathway. Wang et al (31) reported that sitagliptin down-regulated the level of Smad3 phosphorylation by inhibiting TGF- $\beta_{1}$, and up-regulated Smad7 protein expression to prevent the production of collagen IV and fibronectin and to reduce extracellular matrix accumulation. Their data inferred that sitagliptin performs anti-fibrosis and anti-inflammatory functions in the kidney.

With respect to blood glucose and lipid levels, sitagliptin was found to reduce TC level but appeared to have no effect on FBG, PBG, HbAlc, or TG. Hence, the role of sitagliptin in decreasing blood glucose levels remains unclear, which is inconsistent with prevailing viewpoints that sitagliptin reduces glucose levels, does not elicit weight gain, and decreases the risk of hypoglycemia $(32,33)$. 
There are some possible reasons for the discrepancies found in the literature. First, the number of included articles regarding PBG and TG was less than 10, so the funnel plot could not evaluate the publication bias. Second, although there is no publication bias in FBG and HbAlc, as shown by Egger's test and asymmetry in the funnel plots, we believe that other biases may have existed in the included studies. Potential biases in the trials that could have led to incorrect conclusions regarding sitagliptin include non-disclosure of allocation concealment, incomplete outcome data, or double-blind analysis status. Only three of five trials that investigated adverse events mentioned these potential biases. As conclusions concerning safety could not be made in our meta-analysis, the safety of sitagliptin requires more rigorous investigations in future clinical trials.

Sitagliptin was shown to reduce proteinuria, ameliorate renal function, and produce an anti-inflammatory effect in patients with early DN. These findings can be useful for guidance in the clinical application of sitagliptin.

\section{Acknowledgements}

Not applicable.

\section{Funding}

The present study was supported by grants from the National Natural Science Foundation of China (grant no. 81573911). The grantee, JY, was responsible for this article.

\section{Availability of data and materials}

All data generated or analyzed during this study are included in this published article.

\section{Authors' contributions}

WL and JY were involved in conceptualization of the study and writing of the manuscript. NL and LW curated the data. WX and LW performed analysis of the data. WX was involved in acquisition of funding. WL reviewed and edited the manuscript. WL and QY supervised the study. WX and LW provided the resources. WL and QY performed the electronic searches on the PubMed, OVID, Cochrane library, Chinese National Knowledge Infrastructure and Wanfang databases. $\mathrm{NL}$ and JY assessed the quality of evidence and participated in outlining the inclusion criteria and exclusion criteria. NL and WL provided final approval of all procedures performed in the present study.

\section{Ethics approval and consent to participate}

Not applicable.

\section{Patient consent for publication}

Not applicable.

\section{Competing interests}

The authors declare that they have no competing interests.

\section{References}

1. Unger RH and Orci L: The essential role of glucagon in the pathogenesis of diabetes mellitus. Lancet 1: 14-16, 1975.

2. Tuttle KR, Bakris GL, Bilous RW, Chiang JL, de Boer IH, Goldstein-Fuchs J, Hirsch IB, Kalantar-Zadeh K, Narva AS, Navaneethan SD, et al: Diabetic kidney disease: A report from an ADA Consensus Conference. Diabetes Care 37: 2864-2883, 2014.

3. Ahn JH, Yu JH, Ko SH, Kwon HS, Kim DJ, Kim JH, Kim CS, Song KH, Won JC, Lim S, et al: Prevalence and determinants of diabetic nephropathy in Korea: Korea national health and nutrition examination survey. Diabetes Metab J 38: 109-119, 2014.

4. Venkatachalam MA, Weinberg JM, Kriz W and Bidanai AK: Failed tubule recovery, AKI-CKD transition, and kidney disease progression. J Am Soc Nephrol 26: 1765-1776, 2015.

5. Reidy K, Kang HM, Hostetter T and Susztak K: Molecular mechanisms of diabetic kidney disease. J Clin Invest 124: 2333-2340, 2014

6. Lee M and Rhee MK: Sitagliptin for type 2 diabetes: A 2015 update. Expert Rev Cardiovasc Ther 13: 597-610, 2015.

7. Fujita H, Taniai H, Murayama H, Ohshiro H, Hayashi H, Sato S, Kikuchi N, Komatsu T, Komatsu K, Komatsu K, et al: DPP-4 inhibition with alogliptin on top of angiotensin II type 1 receptor blockade ameliorates albuminuria via up-regulation of SDF-1 $\alpha$ in type 2 diabetic patients with incipient nephropathy. Endocr J 61: 159-166, 2014.

8. Hattori S: Sitagliptin reduces albuminuria in patients with type 2 diabetes. Endocr J 58: 69-73, 2011.

9. Kawasaki I, Hiura Y, Tamai A, Yoshida Y, Yakusiji Y, Ikuno Y, Okada M, Ueno H, Tanaka N, Yamagami K, et al: Sitagliptin reduces the urine albumin-to-creatinine ratio in type 2 diabetes through decreasing both blood pressure and estimated glomerular filtration rate. J Diabetes 7: 41-46, 2015.

10. Mistry GC, Maes AL, Lasseter KC, Davies MJ, Gottesdiener KM, Wagner JA and Herman GA: Effect of sitagliptin, a dipeptidyl peptidase-4 inhibitor, on blood pressure in nondiabetic patients with mild to moderate hypertension. J Clin Pharmacol 48: 592-598, 2008.

11. Ogawa S, Ishiki M, Nako K, Okamura M, Senda M, Mori T and Ito S: Sitagliptin, a dipeptidyl peptidase-4 inhibitor, decreases systolic blood pressure in Japanese hypertensive patients with type 2 diabetes. Tohoku J Exp Med 223: 133-135, 2011.

12. Gutzwiller JP, Tschopp S, Bock A, Zehnder CE, Huber AR, Kreyenbuehl M, Gutmann H, Drewe J, Henzen C, Goeke B and Beglinger C: Glucagon-like peptide 1 induces natriuresis in healthy subjects and in insulin-resistant obese men. J Clin Endocrinol Metab 89: 3055-3061, 2004.

13. Higgins JPT and Green S (eds): Cochrane handbook for systematic reviews of interventions version 5.1.2 [updated March 2011]. The Cochrane Collaboration, 2011. Available from: http://handbook.cochrane.org/accessed April 8, 2018.

14. Mori H, Okada Y, Arao T and Tanaka Y: Sitagliptin improves albuminuria in patients with type 2 diabetes mellitus. J Diabetes Investig 5: 313-319, 2014.

15. Zhang K, Ren Q, Wu T and Du J: Effects of sitagliptin used alone or combined with irbesartan on changes of interlukin-18 in early diabetic nephropathy patients. Med J Air Force 1: 56-58, 2016 (In Chinese).

16. Hu S, Bai X, Li X, Li S and Lv S: Effect of sitagliptin on microalbuminuria of patients with type 2 diabetes mellitus. Prog Mod Biomed 16: 5324-5326, 2016 (In Chinese).

17. Huang J, Xu H, Lin Y, Lin B, Wei Y, Yuan B and Chen X: Effect of sitagliptin in combination with atorvastatin on urinary albumin-to-creatinine ratio in type 2 diabetic patients with early nephropathy. Acad J Guangzhou Med Univ 44: 51-53, 2016 (In Chinese).

18. Jin J, Sun H, Xu Z, Lin Z and Tao K: Sitagliptin influence on clinical indicators in elderly patients with diabetic nephropathy. Chin J Gerontol 36: 79-81, 2016 (In Chinese)

19. Lan L and Yan Z: Protective effects of DPP-4 inhibitors on kidney in patients with type 2 diabetes. Chin Foreig Med Res 14: 1-3, 2016 (In Chinese).

20. Yang T, Li D, Zhao J, Liu L and Hu L: Effects of sitagliptin on renal function in patients with early type 2 diabetic nephropathy. Prog Mod Biomed 19: 3690-3693, 2015 (In Chinese).

21. Ying J, Zhang X, Chen J and Chen G: Effects of sitagliptin on renal function and gamma-glutamyltransferase in diabetes combined with kidney injury. Chin J New Drugs Clin Rem 35: 273-276, 2016 (In Chinese). 
22. Zhao C, Guo H, Dai H, Tian J and Zhao Y: Curative effects of sitagliptin with valsartan on incipient type 2 diabetic nephropathy. Pract Pharm Remed 11: 1420-1423, 2014 (In Chinese).

23. Huang T: The effects of urine PCX excretion for type 2 diabetic patients with early nephropathy with treatment of sitagliptin. Shanxi Med Univ: 7-10, 2016 (In Chinese).

24. Wang X, Zhang H, Zhang X, Zhang R, Shi M, Lyu S and Gao P: Effects of sitagliptin on serum chemerin in early diabetic patients with nephropathy. Acta Acad Med Xuzhou 35: 825-828, 2015 (In Chinese).

25. Han H: Sitagliptin for type 2 diabetes early kidney disease curative effect observation. Shanxi Med Univ: 5-9, 2015 (In Chinese).

26. Hao Q, Liu Y and Fei D: The effect of sitagliptin on type 2 diabetes mellitus complicated with early diabetic kidney disease patients. J Taishan Med Coll 36: 989-991, 2015 (In Chinese).

27. Aschner P, Chan J, Owens DR, Picard S, Wang E, Dain MP, Pilorget V, Echtay A and Fonseca V; EASIE investigators: Insulin glargine versus sitagliptin in insulin-naive patients with type 2 diabetes mellitus uncontrolled on metformin (EASIE): A multicentre, randomised open-label trial. Lancet 379: 2262-2269, 2012

28. Katsuno T, Ikeda H, Ida K, Miyagawa J and Namba M: Add-on therapy with the DPP-4 inhibitor sitagliptin improves glycemic control in insulin-treated Japanese patients with type 2 diabetes mellitus. Endocr J 60: 733-742, 2013.

29. Lopez-Giacoman S and Madero M: Biomarkers in chronic kidney disease, from kidney function to kidney damage. World J Nephrol 4: 57-73, 2015.
30. Javanmardi M, Azadi NA, Amini S and Abdi M: Diagnostic value of cystatin $\mathrm{C}$ for diagnosis of early renal damages in type 2 diabetic mellitus patients: The first experience in Iran. J Res Med Sci 20: 571-576, 2015.

31. Wang D, Zhang G, Chen X, Wei T, Liu C, Chen C, Gong Y and Wei Q: Sitagliptin ameliorates diabetic nephropathy by blocking TGF- $\beta 1 /$ Smad signaling pathway. Int J Mol Med 41: 2784-2792, 2018.

32. Tsurutani Y,Omura M, Matsuzawa Y,Saito J,Higa M, Taniyama M and Nishikawa T; SINGLE-Y investigation group: Efficacy and safety of the dipeptidyl peptidase-4 inhibitor sitagliptin on atherosclerosis, $\beta$-cell function, and glycemic control in Japanese patients with Type 2 diabetes mellitus who are treatment naïve or poorly responsive to antidiabetes agents: A multicenter, prospective observational, uncontrolled study. Curr Ther Res Clin Exp 84: 26-31, 2017.

33. Arjona Ferreira JC, Marre M, Barzilai N, Guo H, Golm GT, Sisk CM, Kaufman KD and Goldstein BJ: Efficacy and safety of sitagliptin versus glipizide in patients with type 2 diabetes and moderate-to-severe chronic renal insufficiency. Diabetes Care 36: 1067-1073, 2013.

This work is licensed under a Creative Commons Attribution-NonCommercial-NoDerivatives 4.0 International (CC BY-NC-ND 4.0) License. 